\title{
Trültzsch-Wijnen, Christine W.: Medienhandeln zwischen Kompetenz, Performanz und Literacy.
}

\author{
Wiesbaden: Springer VS 2020. 586 Seiten. Preis: $€ 49,99$
}

\author{
Johannes Gemkow
}

Angenommen: 6. März 2021 / Online publiziert: 23. März 2021

(C) Der/die Autor(en) 2021

Die vorliegende Monografie zeichnet ein breites theoretisches Bild des individuellen Medienhandelns von Kindern und Jugendlichen. Der Fokus liegt auf der Medienkompetenz und insbesondere auf den individuellen und sozialen Determinanten kompetenten Medienhandelns. Den Schwerpunkt des Buches bildet die theoretische Konzeptualisierung und Zusammenführung der heterogenen Begriffe Medienhandeln, Medienaneignung, Mediensozialisation, Medienkompetenz, Medienperformanz und Media Literacy. Dafür greift die Autorin auf grundlegende theoretische Ansätze zurück. Das Buch bleibt aber nicht bei einer theoretischen Diskussion stehen, sondern liefert dem Leser zwei Studien, die die theoretischen Überlegungen empirisch unterfüttern. Die erste Studie ist ein sekundäranalytisches Aktionsforschungsprojekt zum Thema Model-Castingshows, welches beginnend im Jahr 2010 von der Autorin durchgeführt worden ist. Die zweite Studie hat ein triangulatives Mixed-Method-Design und bietet einen quantitativen Einblick in die Social-WebNutzung von Kindern, Jugendlichen und jungen Erwachsenen im Alter von zehn bis dreißig Jahren. Weiterhin bietet es eine qualitative Auseinandersetzung mit den Konzepten von Privatheit und Öffentlichkeit im Umgang mit dem Social Web und den Fähigkeiten, Fertigkeiten und individuellen Handlungsstrategien Heranwachsender. Die qualitativen Ergebnisse werden durch dieses Buch erstmalig publiziert. Das Buch zeigt differenzierte, da theoretisch breite und empirisch hergeleitete, Befunde zur individuellen und sozialen Determiniertheit kompetenten Medienhandelns.

Das Buch hat einen breiten sozialwissenschaftlichen Ansatz. Während sich das erste Kapitel vor allem auf subjektivistische Grundlagen, wie Aneignung, Psychologie und Sozialisation stützt, geht das zweite Kapitel mit Bourdieus Habitustheorie

\footnotetext{
Dr. J. Gemkow $(\bowtie)$

Professur für Medienkompetenz- und Aneignungsforschung, Universität Leipzig,

Emil-Fuchs-Straße 1, 04105 Leipzig, Deutschland

E-Mail: johannes.gemkow@uni-leipzig.de
} 
und Kapitalbegriff stärker auf soziale Grundlagen ein. Mit der Diskussion der Begriffe Kompetenz, Performanz und Literacy wird in den anschließenden Kapiteln der medienpädagogische Impetus des Buches deutlich. Die Zielgruppe des Buches liegt somit insbesondere in der Medienpädagogik und darüber hinaus bei sozialwissenschaftlich Interessierten, die sich mit der Verknüpfung von sozialem Umfeld und Medienhandeln beschäftigen. Die verständliche und grundlagentheoretische Herangehensweise eignet sich hervorragen für Studierende und Personen, die sich in das Thema erstmals einlesen wollen.

Das Buch ist in seinem Überblicks- und Zusammenführungscharakter originell. Die Betitelung des Buches mit Performanz und Literacy ließe zwar vermuten, dass diesen Begriffen noch stärker nachgegangen wird. Das geschieht jedoch nicht. Auch ein theoretisches Verständnis, warum diese Begriffe nun ins Feld geführt werden, vermisst der Leser. Die Ansätze zu Kompetenz, fernab von subjekt-idealistischen Fähigkeiten, zeigen jedoch, dass das Buch mit der Betrachtung individueller und sozialer Determiniertheiten über den ursprünglichen, aber mittlerweile auch zweifelhaften, Common Sense zur Medienkompetenz hinausgeht.

Obgleich das hermeneutische und analysierende Design die Ziele der Arbeit bedient, bleibt eine methodologische Diskussion außen vor. Interessant wäre insbesondere die Verzahnung von subjektivistischen Theorien, wie Medienaneignung, Medienhandeln und Medienkompetenz mit dem eher sozial determinierten Habitus von Bourdieu gewesen. Das Unterfangen, die theoretische Konzeptualisierung empirisch beispielhaft anzuwenden, ist für den Leser besonders interessant. Das Buch liefert darauf aufbauend wichtige Marksteine für individuelle und soziale Determinanten, die sich auf verschiedene Sozialisationsinstanzen berufen. Der Leser muss sich dabei aber im Klaren sein, dass die empirischen Studien gerade nicht stringent aus dem Forschungsziel des Buches abgeleitet sind, sondern nur beispielhaft dessen Relevanz unterstützt. Die konsequente und wissenschaftlich hochwertige Auseinandersetzung mit sehr heterogenen und breit rezipierten Begriffen ist bemerkenswert. Eine (visuelle) Modellierung der diskutierten Begriffe bleibt dabei aus.

Das Buch steht beispielhaft für den Wandel der Medienpädagogik von einer rein subjektivistischen hin zu einer ganzheitlichen Disziplin, die auch individuelle, soziale und, wenn im Buch auch nicht besprochen, mediale Determinanten zu ihrem wissenschaftlichen Repertoire zählt. Das Buch wird als Querschnittsarbeit zwischen Medienhandeln, Medienkompetenz und Mediensozialisation von Kindern und Jugendlichen vor allem Orientierungscharakter haben. Insbesondere die heterogenen theoretischen Konzepte und deren exemplarische empirische Anwendung haben hohe Relevanz für den Bereich Medienpädagogik.

Funding Open Access funding enabled and organized by Projekt DEAL.

Open Access Dieser Artikel wird unter der Creative Commons Namensnennung 4.0 International Lizenz veröffentlicht, welche die Nutzung, Vervielfältigung, Bearbeitung, Verbreitung und Wiedergabe in jeglichem Medium und Format erlaubt, sofern Sie den/die ursprünglichen Autor(en) und die Quelle ordnungsgemäß nennen, einen Link zur Creative Commons Lizenz beifügen und angeben, ob Änderungen vorgenommen wurden.

Die in diesem Artikel enthaltenen Bilder und sonstiges Drittmaterial unterliegen ebenfalls der genannten Creative Commons Lizenz, sofern sich aus der Abbildungslegende nichts anderes ergibt. Sofern das betref- 
fende Material nicht unter der genannten Creative Commons Lizenz steht und die betreffende Handlung nicht nach gesetzlichen Vorschriften erlaubt ist, ist für die oben aufgeführten Weiterverwendungen des Materials die Einwilligung des jeweiligen Rechteinhabers einzuholen.

Weitere Details zur Lizenz entnehmen Sie bitte der Lizenzinformation auf http://creativecommons.org/ licenses/by/4.0/deed.de.

Dr. Johannes Gemkow ist wissenschaftlicher Mitarbeiter an der Professur für Medienkompetenz- und Aneignungsforschung der Universität Leipzig und arbeitet für das Forschungsinstitut Gesellschaftlicher Zusammenhalt (FGZ). 